\title{
Correction to: Machine learning misclassification of academic publications reveals non-trivial interdependencies of scientific disciplines
}

\author{
Alexey Lyutov ${ }^{1} \mathbb{D} \cdot$ Yilmaz Uygun ${ }^{1} \cdot$ Marc-Thorsten Hütt ${ }^{2}$
}

Published online: 28 January 2021

(c) Akadémiai Kiadó, Budapest, Hungary 2021

\section{Correction to: Scientometrics https://doi.org/10.1007/s11192-020-03789-8}

In the original publication of the article, two of the reference citations were incorrectly published. These have been corrected with this Correction as below:

In the Introduction section, the paragraph beginning, "Here we analyze all papers published in PNAS...", the sentence with the reference citation should read as "In addition, directly after the initial data acquisition procedure, the corresponding citations count was obtained for each paper using the Scopus database (Scopus https://dev.elsevier.com/).

In the Results section, the paragraph beginning, "In the beginning, we analyze the variation...", the sentence with the reference citation should read as "Scientific abstracts usually have a typical structure, as described, for example, in the "Nature guide to authors" (Nature https://www.nature.com/documents/nature-summary-paragraph.pdf) or in the traditional hourglass model (Derntl 2014)."

The original article has been corrected.

The original article can be found online at https://doi.org/10.1007/s11192-020-03789-8.

Alexey Lyutov

a.lyutov@jacobs-university.de

1 Department of Mathematics and Logistics, Jacobs University, Campus Ring 1, 28759 Bremen, Germany

2 Department of Life Sciences and Chemistry, Jacobs University, Campus Ring 1, 28759 Bremen, Germany 